\title{
Characterization of the upper respiratory tract microbiome of turkeys
}

Olimpia Kursa ( $\nabla$ olimpia.kursa@piwet.pulawy.pl )

National Veterinary Research Institute https://orcid.org/0000-0002-0058-1568

Grzegorz Tomczyk

Panstwowy Instytut Weterynaryjny - Panstwowy Instytut Badawczy w Pulawach

Anna Sawicka-Durkalec

Panstwowy Instytut Weterynaryjny - Panstwowy Instytut Badawczy w Pulawach

Aleksandra Giza

Panstwowy Instytut Weterynaryjny - Panstwowy Instytut Badawczy w Pulawach

Magdalena Słomiany-Szwarc

Panstwowy Instytut Weterynaryjny - Panstwowy Instytut Badawczy w Pulawach

\section{Research}

Keywords: upper respiratory tract, microbiome, 16S rRNA, turkeys

Posted Date: June 10th, 2020

DOI: https://doi.org/10.21203/rs.3.rs-33858/v1

License: (c) (i) This work is licensed under a Creative Commons Attribution 4.0 International License. Read Full License 
1 Olimpia Kursa ${ }^{1}$, Grzegorz Tomczyk ${ }^{1}$, Anna Sawicka-Durkalec ${ }^{1}$, Aleksandra Giza ${ }^{2}$, Magdalena

2 Słomiany-Szwarc ${ }^{2}$

3 Characterization of the upper respiratory tract microbiome of turkeys

$4 \quad{ }^{1}$ National Veterinary Research Institute, Department of Poultry Diseases, Al. Partyzantów 57,

5 24-100 Pulawy, Poland, phone: 048818893370

6 Grzegorz Tomczyk - gtomczyk@ piwet.pulawy.pl

7 Anna Sawicka-Durkalec - anna.sawicka@ piwet.pulawy.pl

8 2National Veterinary Research Institute, Department of Omics Analyses, Al. Partyzantów 57,

9 24-100 Pulawy, Poland, phone: 048818893370

10 Aleksandra Giza - aleksandra.giza@piwet.pulawy.pl

11 Magdalena Słomiany-Szwarc - magdalena.slomiany-szwarc@piwet.pulawy.pl

\section{Corresponding author}

Email address: olimpia.kursa@piwet.pulawy.pl 


\section{Abstract}

Background: The respiratory tracts of turkeys are the main route of infection therefore plays important roles in the overall health and performance of the birds. Understanding the poultry microbiome has the potential to offer better diagnosis and rational management of many poultry diseases. Characterization of microbial communities in the upper respiratory tract of turkeys could help better understand the role of pathogenic bacteria and other commensal or symbiotic microorganisms in the infection. The aim of this study was microbiome characterization of upper respiratory tracks of commercial turkeys using next-generation sequencing technologies. Results: The microbiome from samples collected from commercial turkey flocks was determined using 16S rRNA metagenomic approach. Taxonomic analysis of the microbiome was done by of the V3 and V4 regions of 16S rRNA gene (MiSeq, Illumina) amplification. The phylogenetic analysis identified the 10 bacterial phyla in turkey, the most abundant were phyla Firmicutes and Proteobacteria, accounting for $>99 \%$ of all the sequences. The turkey sequences represent 144 established bacterial genera. Differences between bacterial abundances were found at the family and genus level. Several defining markers of microbiome succession were identified, including the presence of Ornithobacterium and Mycoplasma.

Conclusions: Understanding the turkey's respiratory microbiome is very important. Unique informations about microbiome representing members of the four major phyla of the respiratory tract in turkeys was assembled. These results obtained in this study supply information about turkey microbiome and can be useful in controlling, diagnosing and treating commercial turkey flocks. Our study significantly broaden the knowledge of the upper respiratory tract microbiome of turkeys.

Key words: upper respiratory tract, microbiome, 16S rRNA, turkeys 


\section{Background}

Next-generation sequencing has resulted in a marked increase in culture-independent studies characterizing the microbiome of humans and animals [1-6]. Much of these works have been focused on the gut microbiome of humans and other production animals [7-11]. The growing number of studies on the avian microbiome demonstrates the influence of the gastrointestinal and respiratory microbiome on the proper development and efficiency of poultry production. In recent years the studies on the bacterial microbiome of poultry have primarily focused on chickens [12-15]. Particularly focused on the composition and diversity of intestinal microbiome of chickens [11,16]. Several studies have also described the gastrointestinal bacterial community in turkeys [11,17]. Less attention has been given to turkeys and their respiratory microbiome. The stability of the avian respiratory microbiome plays a critical role in preventing the colonization of pathogens. Any disruption of microbiological composition can lead to infection. Infection with bacteria such as mycoplasmas is commonly followed by a secondary bacterial or viral infection, leading to increased morbidity and mortality [18]. Infections such as coryza or infectious laryngotracheitis may be limited to the respiratory system, at least initially [19]. The pathogens could cause chronic subclinical upper respiratory infection. Bacterial species with the potential to induce infections in the respiratory tract, such as Escherichia coli, Ornithobacterium rhinotracheale, are often found in association with $M$. gallisepticum or M. synoviae [20,21]. In some cases, respiratory infections observed in a flock may be a component of a multisystemic disease or it may be the predominant disease with lesser involvement of other organ systems [19].

The microbiome is a bacterial community including commensal, symbiotic and pathogenic microorganisms which usually colonize an area of host affecting his health status [4]. The respiratory tracts of turkeys are colonized by bacteria that play important roles in the overall health and performance of the birds. We explored the bacterial communities of the upper 
respiratory tract of turkeys, which will significantly broaden the knowledge about its composition. This study was conducted to define the core microbiome colonizing the upper respiratory tract (URT) in commercial turkey flocks.

\section{Methods}

\section{Sample collection}

Samples were collected from commercial turkeys from geographically distinct farms. Respiratory samples consisted of pooled trachea swabs that were collected from 60 birds per flock. Most birds at the time of sampling did not display lesions or other respiratory signs of clinical disease, only one flock (T-URT-9) had neurological symptoms and weak respiratory signs. Swab samples were shipped to the National Veterinary Research Institute as part of a monitoring program or for diagnostic tests. Relevant flocks metadata including age, year of sampling and location of flocks are in table 1. All samples were suspended in phosphate buffered saline (PBS) $\left(1 \mathrm{ml}\right.$ PBS per one swab) and stored at $-20^{\circ} \mathrm{C}$. Part of the suspension was centrifuged for $10 \mathrm{~min}$ at $10,000 \mathrm{rpm}$. The supernatant was carefully removed and the pellets were suspended in $800 \mu$ PBS. The supernatant was used for DNA isolation.

Table 1. Flock metadata.

\begin{tabular}{|l|l|l|l|}
\hline ID of sample & Location & Age (week) & Year of sampling \\
\hline T-URT-1 & lubelskie & 3 & 2020 \\
\hline T-URT-2 & lubelskie & 3 & 2020 \\
\hline T-URT-3 & warmińsko-mazurskie & 30 & 2019 \\
\hline T-URT-4 & warmińsko-mazurskie & 52 & 2017 \\
\hline T-URT-5 & warmińsko-mazurskie & 3 & 2017 \\
\hline T-URT-6 & wielkopolskie & 22 & 2017 \\
\hline T-URT-7 & warmińsko-mazurskie & 3 & 2019 \\
\hline T-URT-8 & kujawsko-pomorskie & 8 & 2017 \\
\hline T-URT-9 & kujawsko-pomorskie & 6 & 2019 \\
\hline
\end{tabular}

\section{DNA extraction and 16S rRNA gene sequencing}

Genomic DNA was isolated from each pooled sample with the use of Maxwell RSC PureFood Pathogen Kit (Promega, USA) according to the manufacturer's recommendations. The quantity 
and quality of the DNA was determined using the Nanodrop 1000 system (Thermo Scientific). Briefly, before starting extraction $50 \mu$ lysozyme (10mg/ml, Novazym), $6 \mu 1$ mutanolysin (5KU/ml, Sigma-Aldrich), and $8 \mu 1$ lysostaphin (5g/ml, Sigma-Aldrich) was added to the samples followed by incubation for $45 \mathrm{~min}$ at $37^{\circ} \mathrm{C}$. All $16 \mathrm{~S}$ libraries were prepared using Illumina metataxonomic protocol: "16S Metagenomic Sequencing Library Preparation”. The V3-V4 regions of 16S rRNA gene were amplified using 2x KAPA HiFi Hot Start Ready Mix (Roche) and primers:

16S Amplicon PCR Forward Primer:

\section{5'TCGTCGGCAGCGTCAGATGTGTATAAGAGACAGCCTACGGGNGGCWGCAG}

16S Amplicon PCR Reverse Primer:

5'GTCTCGTGGGCTCGGAGATGTGTATAAGAGACAGGACTACHVGGGTATCTAATC $\mathrm{C}$

The length of targeted region is approximately $460 \mathrm{bp}$. Primers include overhang adapter sequences, which are compatible with Illumina index and sequencing adapters. PCR was conducted according to the manufacturer's recommendations. Products were checked on Fragment Analyzer using kit: dsDNA 935 Reagent Kit. Clean up step was performed using AMPure XP beads (Beckman Coulter), according to the protocol. Index PCR step was carried out with use of 2x KAPA HiFi Hot Start Ready Mix (Roche) and dual Index adapters (Illumina) according to the manufacturer's recommendations. Clean up step was again performed using AMPure XP beads (Beckman Coulter), according to the protocol. Libraries were checked and average libraries sizes were determined on Fragment Analyzer, using kit: dsDNA 935 Reagent Kit. Quantification of the libraries was carried out with use of Qubit 3.0 fluorometer (Thermo Fisher Scientific). Normalization of the libraries was performed according to the protocol, libraries were pooled in equimolar concentration and then denatured according to the Illumina protocol and diluted to the final concentration $20 \mathrm{pmol}$. The diversity of the run was ensured 
by compositing metataxonomic with WGS Illumina libraries. The sequencing run was performed on Illumina MiSeq platform and MiSeq reagent kit V3 (600 cycles) with paired reads.

\section{S rRNA gene taxonomy assignments}

The 16S rRNA gene sequencing data was processed through the Quantitative Insights into Microbial Ecology (QIIME 2Core 2020.2) [22] and Krona [23]. The sequences were clustered into operational taxonomic units (OTUs) using dada2 denoise-paired method with parameters: --p-trim-left $m$ which trims off the first $m$ bases of each sequence (Illumina indexes trimming); --p-trunc-len $n$ which truncates each sequence at position $n$ (forward reads $n=300$, reverse reads $\mathrm{n}=242$ ) allowing to remove regions of sequences below quality score 15 . During this step, reads were also corrected and chimeric sequences filtered. Trained Silva 132 99\% OTUs (full-length) classifier was used to assign taxonomy to sequences. Sampling depth was even to 62,338 to ensure that every sample was taken into consideration in analysis.

To compare and illustrate the overall URT microbial community structures of the turkeys flocks Krona charts that allow comparison between microbiomes based on detailed phylogenetic composition were generated. Krona charts were generated using the - krona_qiime.py from Qiime2_pipeline_IT_EMP.md(https://github.com/lokeshbio/AmpliSeq/blob/master/Qiime2_p ipeline IT EMP.md\#krona-plots). The Venn diagrams, including all OTUs generated by the OTU picking step, were calculated using the website Bioinformatics \& Evolutionary Genomics (http://bioinformatics.psb.ugent.be/webtools/Venn). The metadata of flocks and corresponding taxonomic classifications in Krona charts have been included as Additional files 1 and 2 respectively.

\section{Statistical analysis of the URT microbiome of turkeys}

The relative taxa abundance of the flocks is presented as a mean \% value. Alpha and beta diversity plots were measured using the Shannon indexes. To assess the association between 
the microbial community of the upper respiratory tract of turkeys ANCOM analysis implemented in QIIME 2 was performed on an unweighted UniFrac distance matrix of 9 samples [24]. The Kruskal-Wallis test was used to detect significant differences in richness and diversity between bacterial communities present in URT in turkeys. Construction of heatmaps was performed using the QIIME 2 feature-table plugin. Principal coordinate analysis graphs (PCoA) were constructed to visualize sample clustering by bacterial community composition. A Venn diagram was constructed to reveal bacterial OTUs at the genera level that were unique or shared between different flocks. The OTUs observed in any samples in a flock were counted.

\section{Results}

\section{URT microbiome of turkey}

Metagenomic methods have been used to describe the microbial community structure of URT in turkeys. We characterized microbial communities by sequencing V3-V4 regions of $16 \mathrm{~S}$ rRNA gene. Flocks from nine different commercial farms concurrently in this study were used. The bacterial diversity in the URT were generally similar but in some cases differences in bacterial diversity were noted (Figure 1).

The sequences from turkeys represent 10 different phyla including one unclassified, 68 established bacterial family and 144 genera (Figure1, 2). Over $99 \%$ of the URT microbiota of the turkeys flocks was comprised of Firmicutes $(69,11 \% \pm 20,53 \%)$, Proteobacteria $(26,41 \%$ $\pm 16,90 \%)$, Bacteroidetes $(2,31 \% \pm 2,17 \%)$, Actinobacteria $(2,26 \% \pm 4,94 \%)$, Tenericutes $(0,015 \% \pm 0,03 \%)$, Cyanobacteria $(0,087 \% \pm 0,218 \%)$ and unclassified phylum $(0,002 \% \pm$ 0,004\%). In one flock (T-URT-1) a very small number of bacteria belonging to phylum Patescibacteria $(0,003 \%)$ was found. However, in another flock - T-URT-2 was also a small number of bacteria from two different phyla: Synergistetes $(0,01 \%)$ and Verrucomicrobia $(0,01 \%)$. The microbiomes of T-URT-7, T-URT-8 and T-URT-9 have a higher abundance of OTUs from Bacteroidetes and Actinobacteria phylum than others flocks. In addition, the 
microbiome of T-URT-9 was dominated by bacteria from the Firmicutes phylum (Figures 1).

The most common bacterial OTUs in this flock were Enterococcus but also classified OTUs Actinobacter, Psychrobacter, Neisseria and also to the species level: ORT and $M$. gallisepticum.

171 In all flocks Firmicutes was dominated by Bacilli and Clostridia, Proteobacteria was largely 172 represented by Gammaproteobacteria and Alphaproteobacteria. Bacteroidetes consistent only 173 of the class Bacteroidia. Actinobacteria was the most abundant classes in the phylum 174 Actinobacteria. The most common bacterial OTUs found on average in URT samples were Enterococcus (38,78\%), Lactobacillus (12,22\%), Escherichia-Shigella (12,04\%), Pseudomonas (1,3\%) and unclassified Enterobacteriaceae (1,81\%) (Figure 3 and Additional 177 file 1 and 2). By comparing the microbiome datasets between the flocks we identified several 178 bacterial clasess, including Bacilli and Clostridia, that were differentially expressed.

179 Upon examining the microbiome composition of turkeys flocks, we found that in each sample are bacteria belonging to a small number of taxonomic classifications. The tracheal swabs included OTUs classified as Ornithobacterium, Mycoplasma, Gallibacterium, Avibacterium, Staphylococcus and Streptococcus (Table 2, Figure 3). The majority of the reads were not 183 distinguishable to the genera node.

Table 2. Selected OTUs in turkeys URT

\begin{tabular}{|l|l|l|l|l|l|l|}
\hline ID & Mycoplasma & Ornithobacterium & Gallibacterium & Avibacterium & Staphylococcus & Streptococcus \\
\hline T-URT-1 & - & - & - & - & + & - \\
\hline T-URT-2 & - & - & - & - & + & + \\
\hline T-URT-3 & - & + & + & - & + & + \\
\hline T-URT-4 & - & - & + & + & + & + \\
\hline T-URT-5 & - & - & - & - & - & - \\
\hline T-URT-6 & + & + & - & - & - & - \\
\hline T-URT-7 & + & + & - & - & + & - \\
\hline T-URT-8 & - & + & - & - & + & + \\
\hline T-URT-9 & + & + & - & - & + & + \\
\hline
\end{tabular}


In order to evaluate microbiota differences between samples, we analyzed the bdiversity based on unweighted UniFrac for these groups. The UniFrac distance matrix was represented through PCoA (Figure 4). The effects of ORT and Mycoplasmas the occurrence and age on bacterial composition were visualized by PCoA based on the weighted UniFrac distance matrix (Figure 4). PCoA clearly distinct samples based on occurrence of these two pathogens. A Venn diagram was constructed to reveal bacterial OTUs that were unique or shared between different flocks (Figure 4).

The richness of the microbial communities in some flocks was decreasing with the age but flocks did not differ significantly in diversity. The PCoA graph showing that the age of flocks with Mycoplasma OTUs were at 3 to 22 weeks and birds whit ORT OTUs were at 3-30 week (Figure 4, Table 1). The Faith PD phylogenetic diversity based on the geographical localization of flocks was no significant difference between flocks (Kruskal-Wallis Test) (Figure 5).

We performed a composition analysis of microbiome (ANCOM) to identify key genera discriminating the microbiota of turkey flocks. From the all observed OTUs at the genus level, two unclassified Enterococcus $(\mathrm{W}=193),(\mathrm{W}=143)$ and ORT $(\mathrm{W}=160)$ showed a significant difference $(\mathrm{p}<0.05)$ in abundance between the microbiome in the URT in Mycoplasma positive flocks (Figure 6).

\section{Discussion}

Understanding the poultry microbiota has the potential to offer better diagnosis and rational management of many poultry diseases including the use of antibiotics. New sequencing technologies enable us to characterize the turkey's respiratory microbiome without using traditional culturing techniques. Through 16S rRNA gene variable regions analysis of the whole bacterial community from tracheal swabs samples, with possibility detection of pathogens without the need for culture. Some of the respiratory pathogens are carried in the healthy flocks and the factors that cause the switch between carriage and disease (such as inhibition by 
commensal bacteria or intercurrent viral infections) are only partly understood. Most of the current research on the commensal bacteria (microbiota) of poultry has been mainly focused on the gut microbiome of chicken, and less attention has been given to turkeys and respiratory microbiome.

In this study, we tested samples from turkey flocks of different ages and geographically distinct farms. We identified bacterial profiles of URT microbiome of turkey and provided new insights for further identification of novel pathogens in farm flocks.

Based on the results of this study, a number of avian commensals as well as pathogens belonging predominantly to the phyla Firmicutes, Proteobacteria, Bacteroidetes, Actinobacteria, and Tenericutes, have been reported. These are the main phyla in the respiratory tract reported also in domestic and wild birds [25,26], and similarly like those present in the respiratory tract microbiota of other animals [27,28]. At the genus level Lactobacillus, Enterococcus, Escherichia-Shigella, Morganella were most abundant and represented the bacterial genera in the respiratory tract of turkeys. In this study, 68 taxa at the family level were observed (Figure 2, 3). One hundred and forty-four genera were found in the URT of turkeys. Results of the study on the digestive system of chickens and turkeys demonstrated that age and environment appear to play a key role in the initial stages of turkey bacterial microbiome maturation $[17,29]$. In other studies, significant differences in the richness and diversity of respiratory track communities were observed between age groups but in the nasal cavity of chickens [15]. However, the tracheal community composition shifted very gradually as the chicken aged. In this study, tracheal swabs samples were analyzed at the OTU level for specific subsets of OTUs representing geographical localization of sampling and age of birds (Figure 5). The richness of the microbial communities in some flocks was decreasing with the age but flocks did not differ significantly in diversity (AMOVA). It was clear from this analysis that there were shared subsets of OTUs present across all samples, unique subsets that were defining 
of OTUs that were dependent on flocks age. In turkeys, as in chickens, the most common bacteria identified in the respiratory tract were members of the Lactobacillales and along with members of the Enterobacteriales [12,30]. In this study, Lactobacillus and Enterococcus were detected in all URT samples; although their relative abundance was varied in some flocks. Some OTUs displayed a temporal trend and were found more prominently in later aged turkeys, such as those classified as Enterococcus and earlier age such as Lactobacillus.

Core microbiome analysis suggests that the URT microbiome is distinct from gut and litter samples, but seems to be partially reflective of both. The data from this study indicate that the turkey URT microbiome is a combination of exposure to the litter environment and preferential selection for microbes from that environment with the capacity to colonize. Bacteria that have previously been found as members of the turkeys gut microbiota, such as Ruminococcus, Clostridium, Virgibacillus, Blautia and also Brachybacterium, Brevibacterium, Staphylococcus, Corynebacterium and Weissella were also isolated in this study $[11,17]$. They were found in ileum and litter samples, but were absent from cecal samples. Bacteria previously isolated from poultry house air, such as Jeotgalicoccus [31] were also found in the respiratory tract in chickens [12] and now also in URT of turkeys.

We note that flocks T-URT-1 and T-URT-2 contained a more diverse microbiome than the other flocks. The cause of this could be various factors such as housing and environmental conditions, age, or performance stress. In URT of these flocks a small abundance of bacteria from three phyla: Patescibacteria, Verrucomicrobia and Synergistetes were found. The bacteria from the family Akkermansiaceae (phylum Verrucomicrobia) are mucin-degrading bacteria and may be the most prestigious microorganisms among the next-generation probiotics [27]. The bacteria from phylum Synergistetes inhabit a majority of anaerobic environments including animal gastrointestinal tracts, soil, and wastewater treatment plants and they are also present in sites of human diseases such as cysts, and areas of periodontal disease [32]. The Patescibacteria 
phylum is the newly defined superphylum and has been found to be prevalent in groundwater, sediment, lake, different aquifer environments and also in soybean. This superphylum has small genomes and a presumed symbiotic or parasitic lifestyle [33,34]. However, we also do not know the role of bacteria that we found as unclassified at the phylum level, therefore a further study of turkeys' respiratory microbiomes is needed.

Several bacterial taxa identified in these study have been described as positively affecting chicken flock performance. These include Bacteroides, Faecalibacterium, Parabacteroides in the gut; and Bifidobacterium, Corynebacterium, Dietzia, Staphylococcus in the trachea [28]. In another study identified Faecalibacterium as being correlated with enhanced feed conversion rates in chickens Bacteroides have been reported as core components of the poultry microbiota in other studies, and perhaps deserve attention for their possible probiotic capabilities $[11,28,35,36]$.

In the URT of turkeys, potential respiratory pathogens including Avibacterium, Gallibacterium, Mycoplasma, and Ornithobacterium were found (Table 2). Multifactorial respiratory disease in poultry is often associated with bacterial factors including E. coli, ORT, Mycoplasma gallisepticum, M. synoviae are frequently implicated. The most pathogenic bacterial species causing significant diseases of poultry are Mycoplasma gallisepticum (mycoplasmosis) and ORT (respiratory disease) [25]. The upper respiratory tract is a reservoir of opportunistic pathogens, which can proliferate and infect the air sacs when poultry immune system is compromised due to stress or primary viral infections. Interestingly, most birds at the time of sampling did not display respiratory signs of clinical disease. One flock T-URT-9 had neurological symptoms and weak respiratory signs. Such neurological problems have been reported in turkeys due to meningoencephalitis caused by MG neurotropic strains [37]. Presents of ORT can also induce osteomyelitis of the cranial bones in turkeys causing nervous signs, movement disturbances and recumbence [38]. This highlights the potential importance of these 
pathogens in the environment, causing also subclinical disease and subsequently impacting the performance of birds. Community level similarities in the bacterial microbiome of samples were compared using principal coordinates analysis (Figure 4). Samples were stratified primarily by the age of flock. Using AMOVA based upon distance matrix, bacterial communities from all sample types were distinct $(\mathrm{P}<0.001)$. Upon visualization of the PCoA plots, Mycoplasma positive and ORT positive samples did not overlap and were distinct from each other. However, all sample types shifted similarly over time on the plot, indicating that bird age has an impact on the bacterial microbiome. Venn diagram showing the number of OTUs at the genus level that were unique or shared between flocks with and without Mycoplasma and Ornithobacterium. Therefore, the interaction between turkeys and these species should be studied to understand the function of the respiratory tract microbiota of turkeys.

Our study identified bacterial profiles of turkeys respiratory microbiome and provided new insights for further identification of novel pathogens in flocks of these birds species. We determined that in the URT turkey microbiome were OTUs of different bacterial species. Most of the organisms not previously associated with the respiratory tracts of birds were present in relatively low proportions. However, it is worth noting that health problems in flocks of turkeys can be caused not only by known pathogens but also by bacteria that were traditionally not considered pathogenic. The avian respiratory tract is the common site of viral pathogen entry and disease, including Newcastle disease, infectious bronchitis, or avian influenza which are dangerous to the health and life of birds. Bacterial infections not only have a devastating effect on the poultry flocks, but they also render flocks immunosuppressed and susceptible to viral infections. Therefore, it is very important for the poultry industry to prevent poultry respiratory infections. To understand the relationship between respiratory tract microbiota of turkeys and factors influencing it, an adequate number of samples will be needed in further study. 


\section{Conclusion}

312 It is very important that various bacteria have been identified in the microbiomes, including 313 potentially pathogenic ones. It underlines the importance of monitoring the microbiome of 314 healthy animals for potential emerging diseases and their treatment. Understanding associations 315 with bacteria present in the airways and potentially pathogenic bacteria highlights the 316 importance of this study.

\section{Abbreviations}

318 URT: Upper respiratory tract

319 QIIME: Quantitative Insights Into Microbial Ecology

320 OTU: Operational taxonomic unit

321 PCoA: Principal Coordinate Analysis

322 Ethics declarations

323 Ethics approval and consent to participate

324 Not applicable

\section{Consent for Publication}

326 Not applicable.

\section{Availability of data and material}

328 All data generated or analysed during this study are included in this published article.

\section{Competing interests}

330 The authors declare that they have no competing interests.

\section{$331 \quad$ Funding}

332 This work was supported by the National Veterinary Research Institute in Puławy, Poland.

\section{Contributions}

OK was involved in study design, performed sample processing, data analysis and preparation of the manuscript draft. GT assisted with the sample organization, data management. AS-D 
assisted with the sample processing, and analyses. AG and MS-S performed sample processing and bioinformatics analyses and were involved in the preparation of the manuscript draft. All authors read and approved the final manuscript.

\section{Acknowledgements}

Not applicable

\section{References}

1. Huttenhower C, Gevers D, Knight R, Abubucker S, Badger JH, Chinwalla AT, et al. Structure, function and diversity of the healthy human microbiome. Nature. Nature Publishing Group; 2012;486:207-14.

2. Wang W, Hu H, Zijlstra RT, Zheng J, Gänzle MG. Metagenomic reconstructions of gut microbial metabolism in weanling pigs. Microbiome. Microbiome; 2019;7:1-11.

3. Wylie KM, Truty RM, Sharpton TJ, Mihindukulasuriya KA, Zhou Y, Gao H, et al. Novel bacterial Taxa in the human microbiome. PLoS One. 2012;7.

4. Sender R, Fuchs S, Milo R. Revised estimates for the number of human and bacteria cells in the body. PLoS Biol. 2016;14.

5. Macovei L, Mccafferty J, Chen T, Teles F, Hasturk H, Paster J, et al. Healthy oral cavity and upper respiratory tract. 2015;2297.

6. Bond SL, Timsit E, Workentine M, Alexander T, Léguillette R. Upper and lower respiratory tract microbiota in horses: Bacterial communities associated with health and mild asthma (inflammatory airway disease) and effects of dexamethasone. BMC Microbiol. BMC Microbiology; 2017;17:1-11.

7. Arumugam M, Raes J, Pelletier E, Paslier D Le, Batto J, Bertalan M, et al. Enterotypes in the landscape of gut microbial community composition. Nature. 2013;3:1-12.

8. Lamendella R, Santo Domingo JW, Ghosh S, Martinson J, Oerther DB. Comparative fecal metagenomics unveils unique functional capacity of the swine gut. BMC Microbiol. 2011;11.

9. Jami E, Mizrahi I. Composition and similarity of bovine rumen microbiota across individual animals. PLoS One. 2012;7:1-8.

10. Best AA, Porter AL, Fraley SM, Fraley GS. Characterization of gut microbiome dynamics in developing pekin ducks and impact of management system. Front Microbiol. 2017;7:1-15. 11. Wei S, Morrison M, Yu Z. Bacterial census of poultry intestinal microbiome. Poult Sci. 2013;92:671-83.

12. Glendinning L, McLachlan G, Vervelde L. Age-related differences in the respiratory 
microbiota of chickens. PLoS One. 2017;12:1-13.

13. Clavijo V, Flórez MJV. The gastrointestinal microbiome and its association with the control of pathogens in broiler chicken production: A review. Poult Sci. 2018;97:1006-21.

14. Choi KY, Lee TK, Sul WJ. Metagenomic analysis of chicken gut microbiota for improving metabolism and health of chickens - A review. Asian-Australasian J. Anim. Sci. 2015.

15. Ngunjiri JM, Taylor KJM, Abundo MC, Jang H, Elaish M, Kc M. Farm stage, bird age, and body site dominantly affect the quantity, taxonomic composition, and dynamics of respiratory and gut microbiota of commercial layer chickens. Appl Environ Microbiol. 2019;85:1-17.

16. Siegerstetter SC, Schmitz-Esser S, Magowan E, Wetzels SU, Zebeli Q, Lawlor PG, et al. Intestinal microbiota profiles associated with low and high residual feed intake in chickens across two geographical locations. PLoS One. 2017;12:1-23.

17. Danzeisen JL, Clayton JB, Huang H, Knights D, McComb B, Hayer SS, et al. Temporal relationships exist between cecum, ileum, and litter bacterial microbiomes in a commercial Turkey flock, and subtherapeutic penicillin treatment impacts ileum bacterial community establishment. Front Vet Sci. 2015;2.

18. Kleven SH. Mycoplasmas in the etiology of multifactorial respiratory disease. Poult Sci. 1998;77:1146-9.

19. Glisson JR. Bacterial Respiratory Diseases of Poultry. Poult Sci [Internet]. Poultry Science Association Inc.; 1998;77:1139-42.

20. Ferguson-Noel N, Noormohammadi AH. Mycoplasma synoviae infection. In: Swayne DE, Glisson JR, McDougald LR, Nolan LK, Suarez DL, Nair VL, editors. Dis Poult. 13th ed. Ames: Wiley; 2013. p. 900-6.

21. Landman WJM. Is Mycoplasma synoviae outrunning Mycoplasma gallisepticum? A viewpoint from the Netherlands. Avian Pathol. 2014;43:2-8.

22. Bolyen E, Rideout JR, Dillon MR, Bokulich NA, Abnet CC, Al-Ghalith GA, et al. Reproducible, interactive, scalable and extensible microbiome data science using QIIME 2. Nat. Biotechnol. 2019. p. 852-7.

23. Ondov BD, Bergman NH, Phillippy AM. Interactive metagenomic visualization in a Web browser. BMC Bioinformatics. BioMed Central Ltd; 2011;12.

24. Lozupone C, Lladser ME, Knights D, Stombaugh J, Knight R. UniFrac: An effective distance metric for microbial community comparison. ISME J. Nature Publishing Group; 2011;5:169-72.

25. Gopala Krishna Murthy TR, Dorairajan N, Balasubramaniam GA, Dinakaran AM, Saravanabava K. Pathogenic bacteria related to respiratory diseases in poultry with reference 
to Ornithobacterium rhinotracheale isolated in India. Vet Arh. 2008;78:131-40.

26. Michiels T, Welby S, Vanrobaeys M, Quinet C, Rouffaer L, Lens L, et al. Prevalence of Mycoplasma gallisepticum and Mycoplasma synoviae in commercial poultry, racing pigeons and wild birds in Belgium. Avian Pathol. 2016;45:244-52.

27. Shabbir MZ, Malys T, Ivanov Y V., Park J, Bakr Shabbir MA, Rabbani M, et al. Microbial communities present in the lower respiratory tract of clinically healthy birds in Pakistan. Poult Sci. 2014;94:612-20.

28. Johnson TJ, Youmans BP, Noll S, Cardona C, Evans NP, Peter Karnezos T, et al. A consistent and predictable commercial broiler chicken bacterial microbiota in antibiotic-free production displays strong correlations with performance. Appl Environ Microbiol. 2018;84. 29. Danzeisen JL, Calvert AJ, Noll SL, McComb B, Sherwood JS, Logue CM, et al. Succession of the turkey gastrointestinal bacterial microbiome related to weight gain. PeerJ. 2013;2013. 30. Sohail MU, Hume ME, Byrd JA, Nisbet DJ, Shabbir MZ, Ijaz A, et al. Molecular analysis of the caecal and tracheal microbiome of heat-stressed broilers supplemented with prebiotic and probiotic. Avian Pathol. 2015;44:67-74.

31. Martin E, Fallschissel K, Kämpfer P, Jäckel U. Detection of Jeotgalicoccus spp. in poultry house air. Syst Appl Microbiol. 2010;33:188-92.

32. Jumas-Bilak E, Carlier JP, Jean-Pierre H, Citron D, Bernard K, Damay A, et al. Jonquetella anthropi gen. nov., sp. nov., the first member of the candidate phylum "Synergistetes" isolated from man. Int J Syst Evol Microbiol. 2007;57:2743-8.

33. Tian R, Ning D, He Z, Zhang P, Spencer SJ, Gao S, et al. Small and mighty: Adaptation of superphylum Patescibacteria to groundwater environment drives their genome simplicity. Microbiome. 2020;8:1-15.

34. Sánchez-Osuna M, Barbé J, Erill I. Comparative genomics of the DNA damage-inducible network in the Patescibacteria. Environ Microbiol. 2017;19:3465-74.

35. Wei S, Lilburn M, Yu Z. The bacteriomes of ileal mucosa and cecal content of broiler chickens and turkeys as revealed by metagenomic analysis. Int J Microbiol. 2016;2016.

36. Oakley BB, Lillehoj HS, Kogut MH, Kim WK, Maurer JJ, Lee MD, et al. The chicken gastrointestinal microbiome. 2014;

37. Wyrzykowski B, Albaric O, Moreau S, Nguyen F, Fleurance R, Belluco S, et al. Retrospective study of Mycoplasma gallisepticum meningoencephalitis in six turkey flocks in western France. J Comp Pathol. Elsevier Ltd; 2013;148:173-7.

38. Moreno B, Chacón G, Villa A, Fernández A, Vela AI, Fernández-Garayzábal JF, et al. Nervous signs associated with otitis and cranial osteomyelitis and with Ornithobacterium 
rhinotracheale infection in red-legged partridges (Alectoris rufa). Avian Pathol. 2009;38:3417.

Figure legends

Fig. 1. Taxonomic diversity plot showing the relative abundance of taxa at the phylum level in each sample.

Fig. 2 Taxonomic diversity plot showing the relative abundance of taxa at the family level in each sample

Fig. 3 Heatmap of bacterial OTUs in turkeys URT

a) Heatmap of bacterial OTUs in the upper respiratory tract of turkeys. Heatmap depicting abundance of all 73 OTUs by overall abundance across samples. Normalized heatmap on taxonomic level 5 was constructed with clustering on both samples and feature axes. b) Shannon diversity index comparing URT flocks.

Fig. 4 Shared and unique OTUs at the genus level in the URT of turkeys.

a) Venn diagram showing the number of OTUs at the genus level in flocks Mycoplasma positive (T-URT-6, T-URT-7, T-URT-9) and flocks Mycoplasma negative (T-URT-3, T-URT-4,TURT-5). b) Venn diagram showing the number of OTUs at the genus level in flocks Mycoplasma positive (T-URT-6, T-URT-7, T-URT-9) and flocks Ornithobacterium positive (T-URT-3, T-URT-6,T-URT-7, T-URT-8). c), d) Clustering of URT of turkeys according to the occurrence of Mycoplasma (c) and ORT (d) in the microbiome. PCoA graph showing the significantly separate clustering by community composition of the bacterial communities in tracheal swabs from turkeys of different ages (AMOVA: $\mathrm{P}<0.001)$.

Fig. 5 Comparison of URT microbiome of turkeys

a) The Faith phylogenetic diversity (PD) rarefaction curve comparing URT microbiome.

b) The Faith PD boxplots. 
Fig. 6 ANCOM Volcano Plot

464 In ANCOM analysis the W value represents the number of times of the null-hypothesis (the 465 average abundance of a given species in a group is equal to that in the other group), it was 466 rejected for a given species. The clr (axe $\mathrm{x})$ is central log ratio.

467

468 Additional file $1-$ KRONA charts

469 Additional file 2 - ID of flocks in Krona file 
Figures

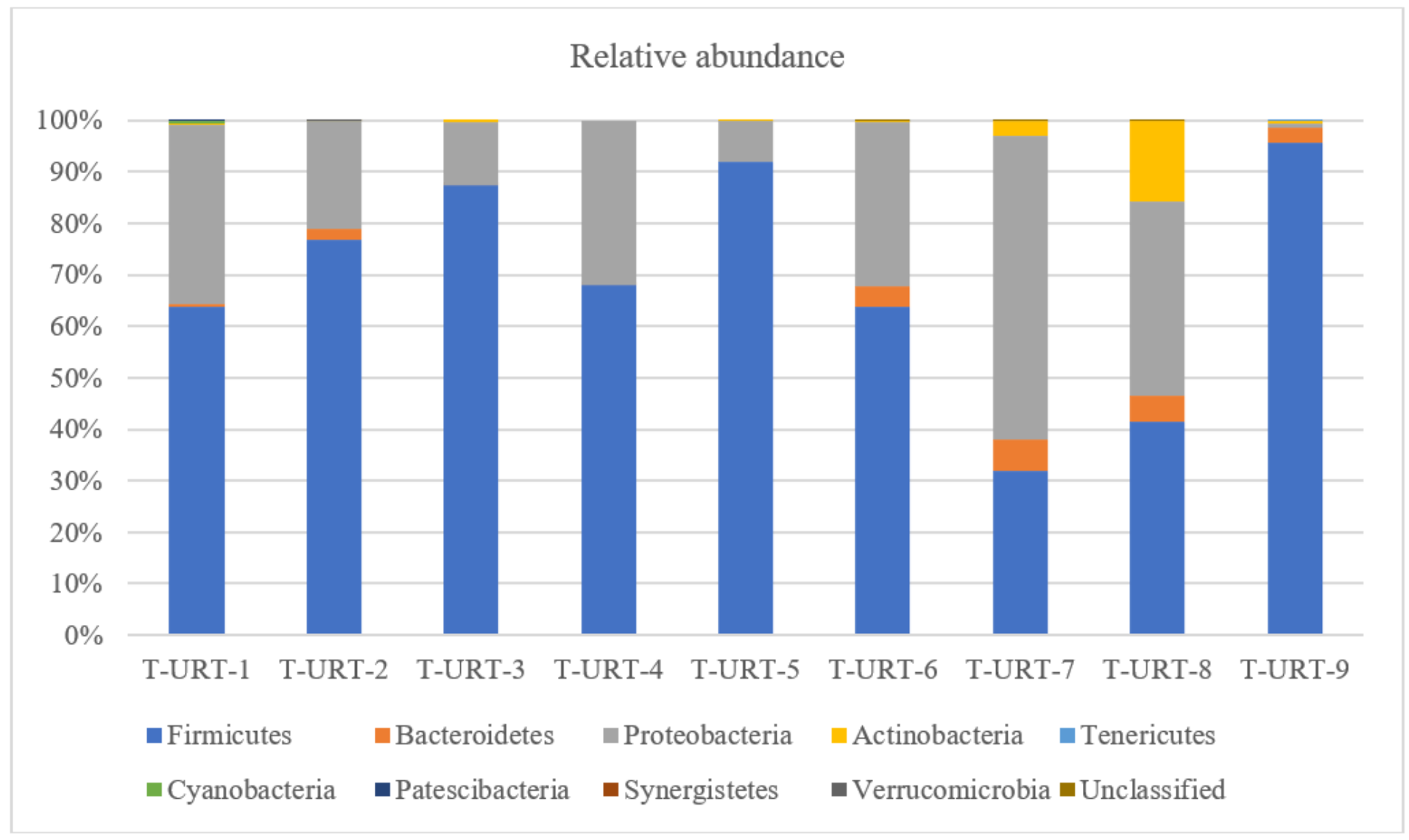

\section{Figure 1}

Taxonomic diversity plot showing the relative abundance of taxa at the phylum level in each sample. 


\section{Relative Abundance}

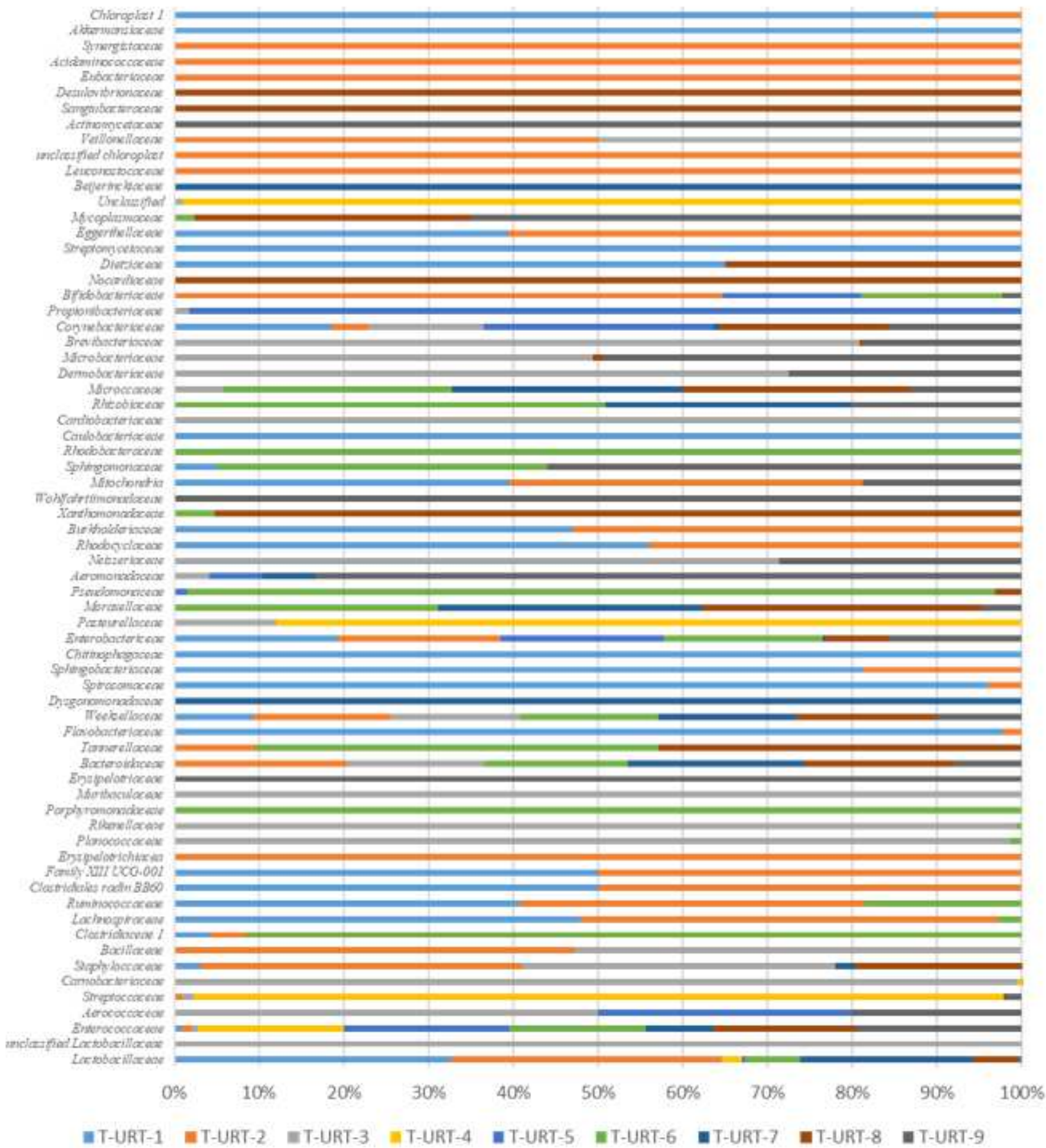

Figure 2

Taxonomic diversity plot showing the relative abundance of taxa at the family level in each sample 

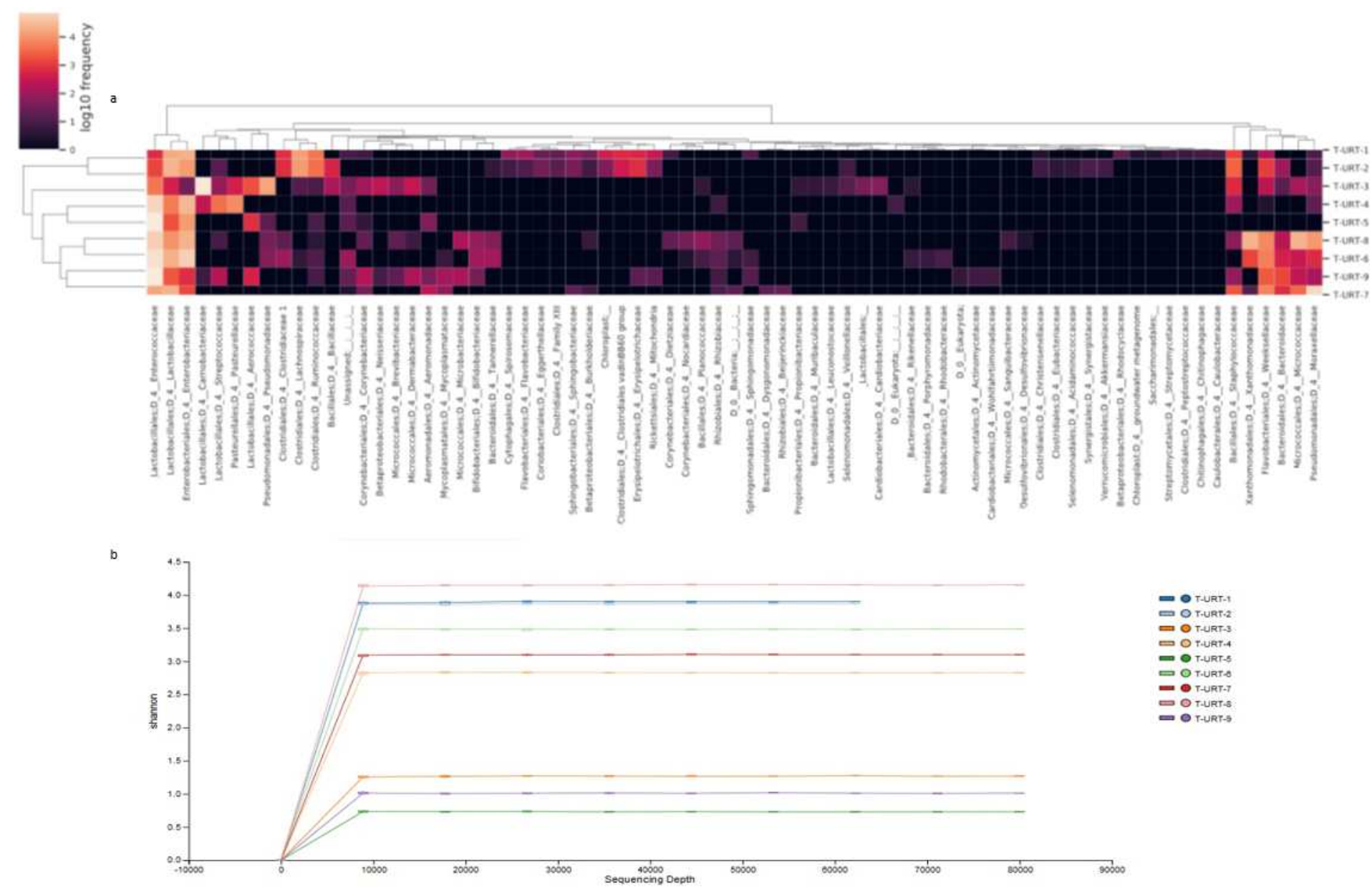

\section{Figure 3}

Heatmap of bacterial OTUs in turkeys URT a) Heatmap of bacterial OTUs in the upper respiratory tract of turkeys. Heatmap depicting abundance of all 73 OTUs by overall abundance across samples. Normalized heatmap on taxonomic level 5 was constructed with clustering on both samples and feature axes. b) Shannon diversity index comparing URT flocks. 


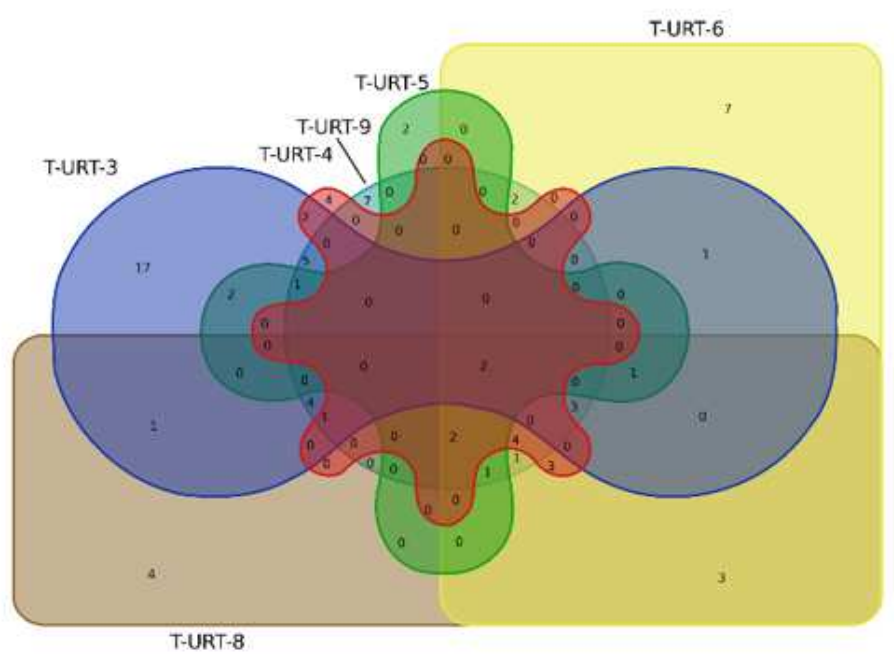

C

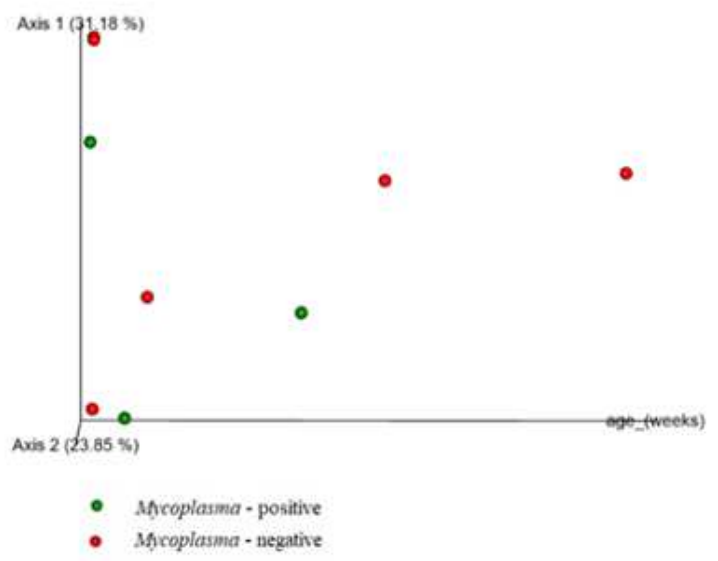

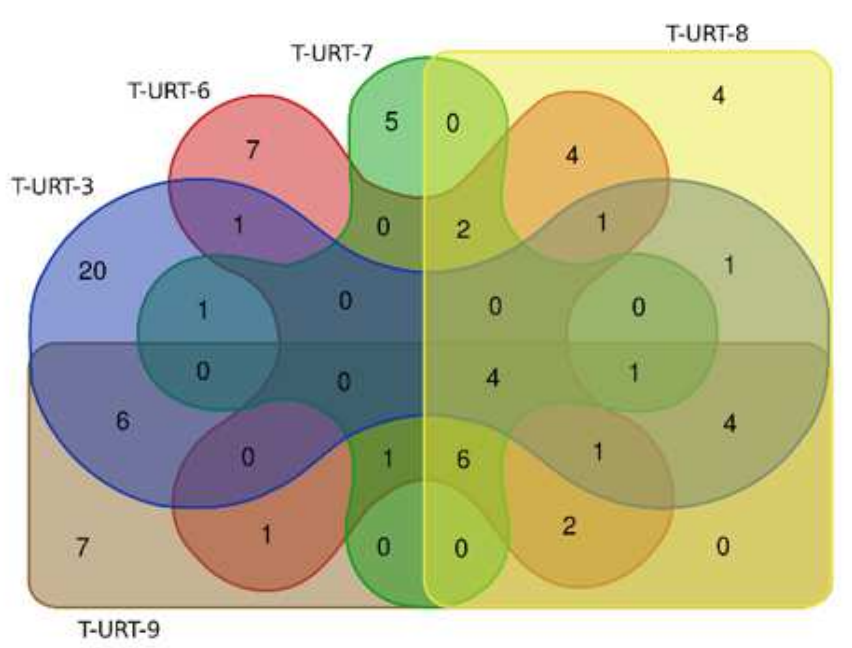

d

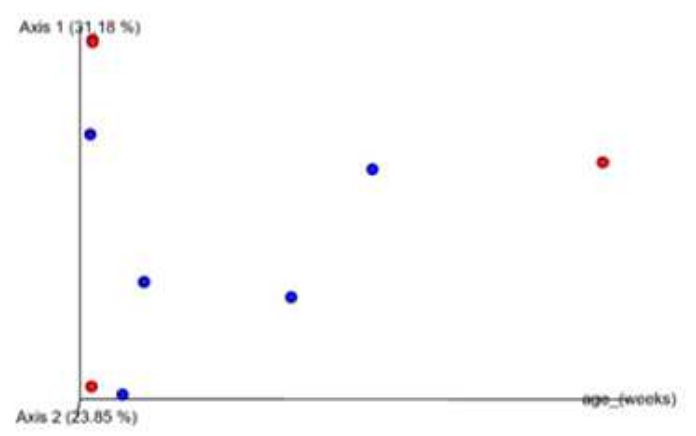

- ORT-positive

\section{Figure 4}

Shared and unique OTUs at the genus level in the URT of turkeys. a) Venn diagram showing the number of OTUs at the genus level in flocks Mycoplasma positive (T-URT-6, T-URT-7, T-URT-9) and flocks Mycoplasma negative (T-URT-3, T-URT-4,T-URT-5). b) Venn diagram showing the number of OTUs at the genus level in flocks Mycoplasma positive (T-URT-6, T-URT-7, T-URT-9) and flocks Ornithobacterium positive (T-URT-3, T-URT-6,T-URT-7, T-URT-8). c), d) Clustering of URT of turkeys according to the occurrence of Mycoplasma (c) and ORT (d) in the microbiome. PCoA graph showing the significantly separate clustering by community composition of the bacterial communities in tracheal swabs from turkeys of different ages (AMOVA: $P<0.001)$. 

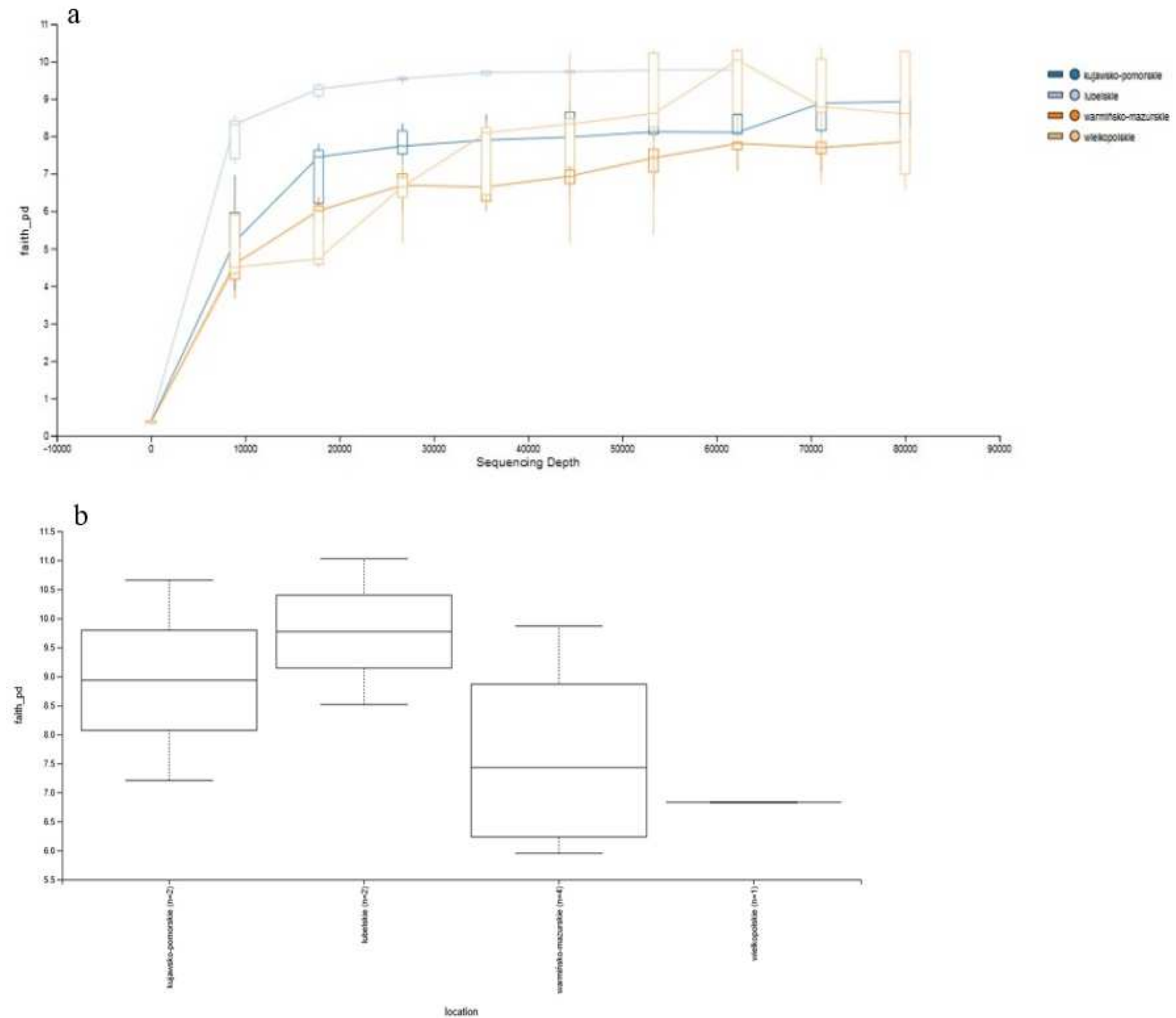

Figure 5

Comparison of URT microbiome of turkeys a) The Faith phylogenetic diversity (PD) rarefaction curve comparing URT microbiome. b) The Faith PD boxplots. 


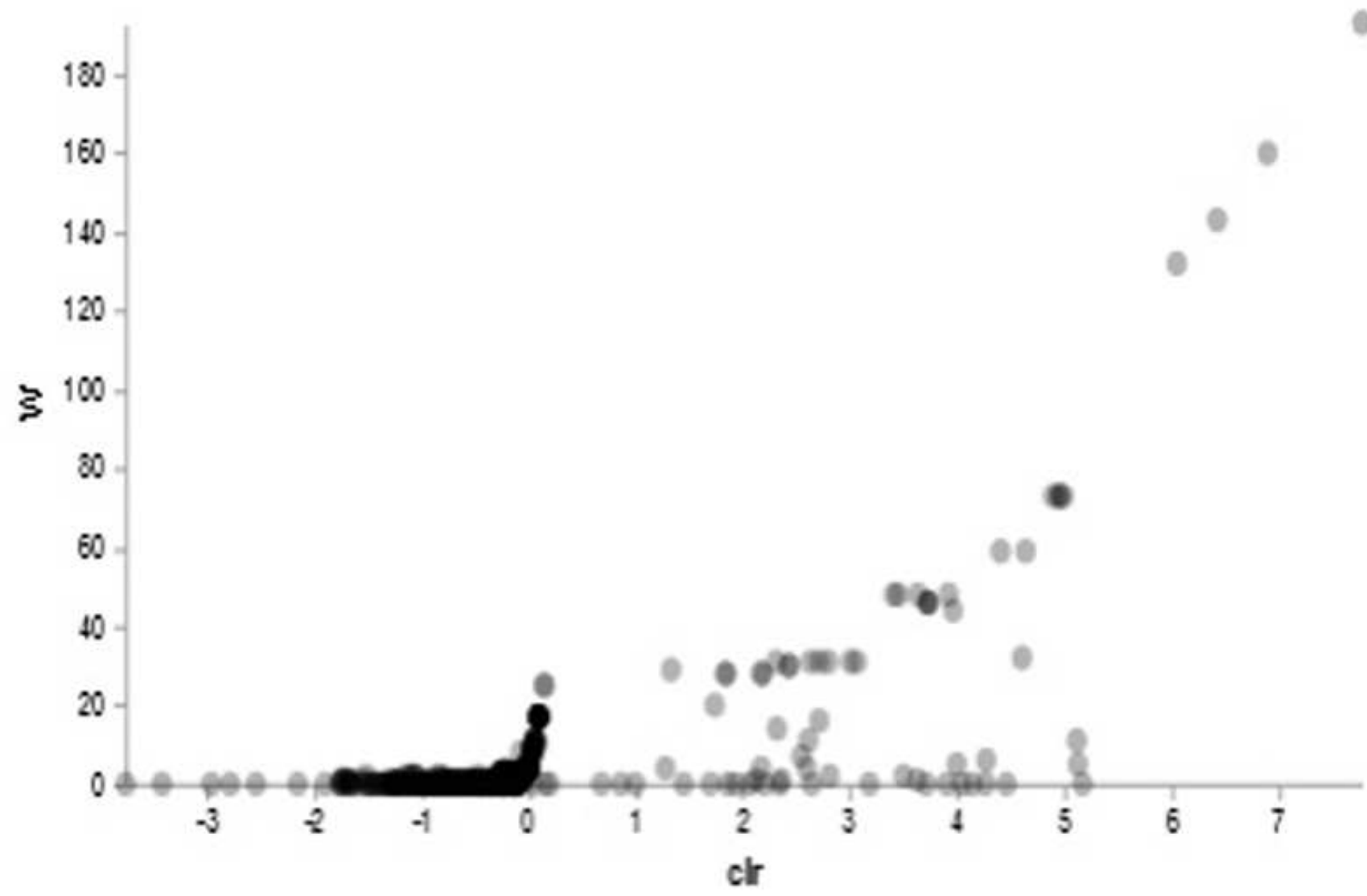

Figure 6

ANCOM Volcano Plot. In ANCOM analysis the $\mathrm{W}$ value represents the number of times of the nullhypothesis (the average abundance of a given species in a group is equal to that in the other group), it was rejected for a given species. The clr (axe $\mathrm{x}$ ) is central log ratio.

\section{Supplementary Files}

This is a list of supplementary files associated with this preprint. Click to download.

- Additionalfile2.docx

- KRONAcharts.html 